\title{
Where There is Smoke There is Fear-Impaired Contextual Inhibition of Conditioned Fear in Smokers
}

\author{
Jan Haaker ${ }^{*, 1,2}$, Tina B Lonsdorf ${ }^{2}$, Dirk Schümann², Nico Bunzeck ${ }^{2,3}$, Jan Peters ${ }^{2,4}$, Tobias Sommer ${ }^{2}$ and \\ Raffael Kalisch ${ }^{2,5,6}$ \\ 'Division of Psychology, Department of Clinical Neuroscience, Karolinska Institutet, Stockholm, Sweden; ${ }^{2}$ Department of Systems Neuroscience, \\ University Medical Center Hamburg-Eppendorf, Hamburg, Germany; ${ }^{3}$ Department of Psychology, University of Lübeck, Germany; ${ }^{4}$ Department \\ of Psychology, Biological Psychology, University of Cologne, Cologne, Germany; ${ }^{5}$ Neuroimaging Center (NIC), Focus Program Translational \\ Neuroscience (FTN), Johannes Gutenberg University Medical Center, Mainz, Germany; ${ }^{6}$ Deutsches Resilienz-Zentrum (DRZ), Johannes Gutenberg \\ University Medical Center, Mainz, Germany
}

\begin{abstract}
The odds-ratio of smoking is elevated in populations with neuropsychiatric diseases, in particular in the highly prevalent diagnoses of post-traumatic stress and anxiety disorders. Yet, the association between smoking and a key dimensional phenotype of these disordersmaladaptive deficits in fear learning and fear inhibition - is unclear. We therefore investigated acquisition and memory of fear and fear inhibition in healthy smoking and non-smoking participants ( $N=349,22 \%$ smokers). We employed a well validated paradigm of contextdependent fear and safety learning (day I) including a memory retrieval on day 2. During fear learning, a geometrical shape was associated with an aversive electrical stimulation (classical fear conditioning, in danger context) and fear responses were extinguished within another context (extinction learning, in safe context). On day 2, the conditioned stimuli were presented again in both contexts, without any aversive stimulation. Autonomic physiological measurements of skin conductance responses as well as subjective evaluations of fear and expectancy of the aversive stimulation were acquired. We found that impairment of fear inhibition (extinction) in the safe context during learning (day I) was associated with the amount of pack-years in smokers. During retrieval of fear memories (day 2), smokers showed an impairment of contextual (safety context-related) fear inhibition as compared with non-smokers. These effects were found in physiological as well as subjective measures of fear. We provide initial evidence that smokers as compared with non-smokers show an impairment of fear inhibition. We propose that smokers have a deficit in integrating contextual signs of safety, which is a hallmark of post-traumatic stress and anxiety disorders.

Neuropsychopharmacology (2017) 42, 1640-1646; doi:I0.1038/npp.20 I7.17; published online I5 February 2017
\end{abstract}

\section{INTRODUCTION}

Smokers are heavily overrepresented among psychiatric patients (Cook et al, 2014; Lasser et al, 2000). In patients suffering from post-traumatic stress disorder (PTSD), the odds-ratio for smoking is between 2 and 4 (Fu et al, 2007) and related to symptom severity in high-risk groups, such as combat veterans (Kirby et al, 2008). Moreover, smoking compromises therapeutic efficiency and enhances relapse risk in PTSD and anxiety disorders after treatment (Taylor et al, 2015). Although several studies observed associations between smoking and categorical diagnoses of PTSD (Breslau et al, 2004; Fu et al, 2007) and other anxietyrelated disorders (Isensee et al, 2003; Lasser et al, 2000), it is unclear if associations between smoking and basic

* Correspondence: Dr J Haaker, Department of Systems Neuroscience, University Medical Center Hamburg-Eppendorf, Hamburg 17177, Germany, Tel: +49 40 74l0 59859, Fax: +49 40 74l0 59955, E-mail: j.haaker@uke.de

Received 18 October 2016; revised 29 November 2016; accepted 10 January 2017; accepted article preview online 25 January 2017 dimensional phenotypes of fear exist. Dimensional measures of fear within the Research Domain Criteria (RDoC) theorem describe the spectrum between adaptive to maladaptive responses within the negative valence systems (ie, lower-order constructs: potential and acute threat Cuthbert and Insel, 2013), where PTSD patients are considered the extreme group on the maladaptive side. An untested hypothesis is that smoking is associated with maladaptive fear responses across the dimensional spectrum in healthy populations.

Dimensional measures of fear responses and fear inhibition can be investigated with pre-clinical models that might provide insights into processes relevant for PTSD. Fear conditioning is the prototypical model for such a purpose where a neutral cue (conditioned stimulus, CS) is associated with an aversive event (unconditioned stimulus, US). In animals, acute nicotine was found to enhance conditioned fear responses (CRs) to CSs and to impair the inhibition of CRs within safe contexts (Kutlu and Gould, 2015). The inability to take advantage of such contextual safety signals may be mechanistically involved in the excessive fear 
a Learning phase (day 1)
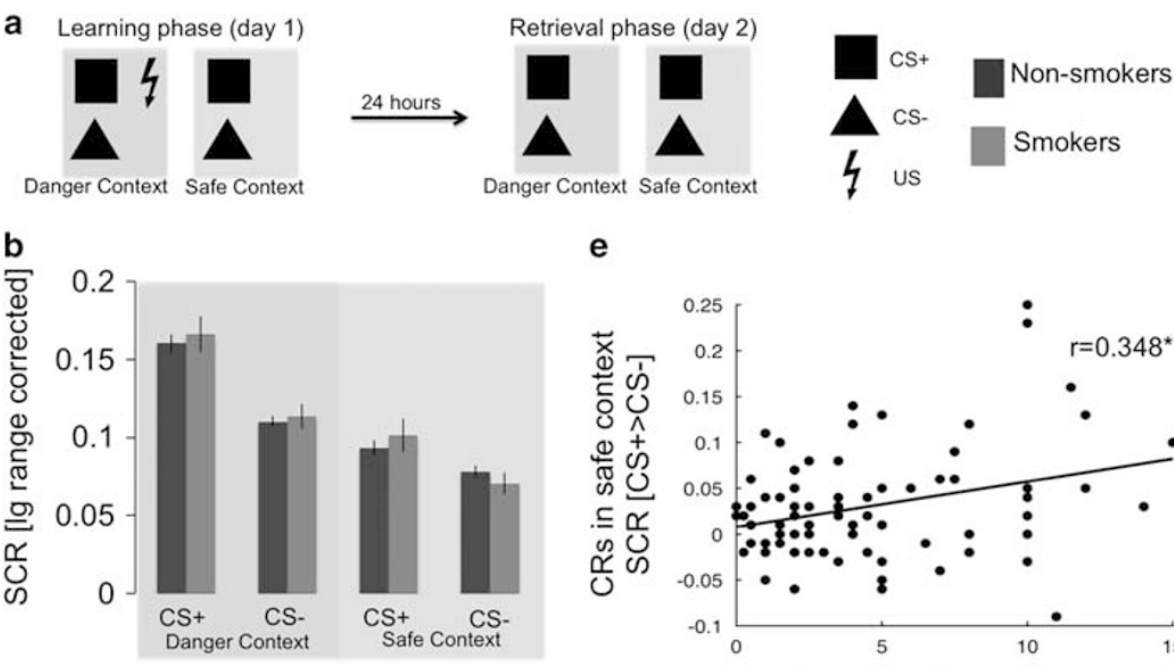

e
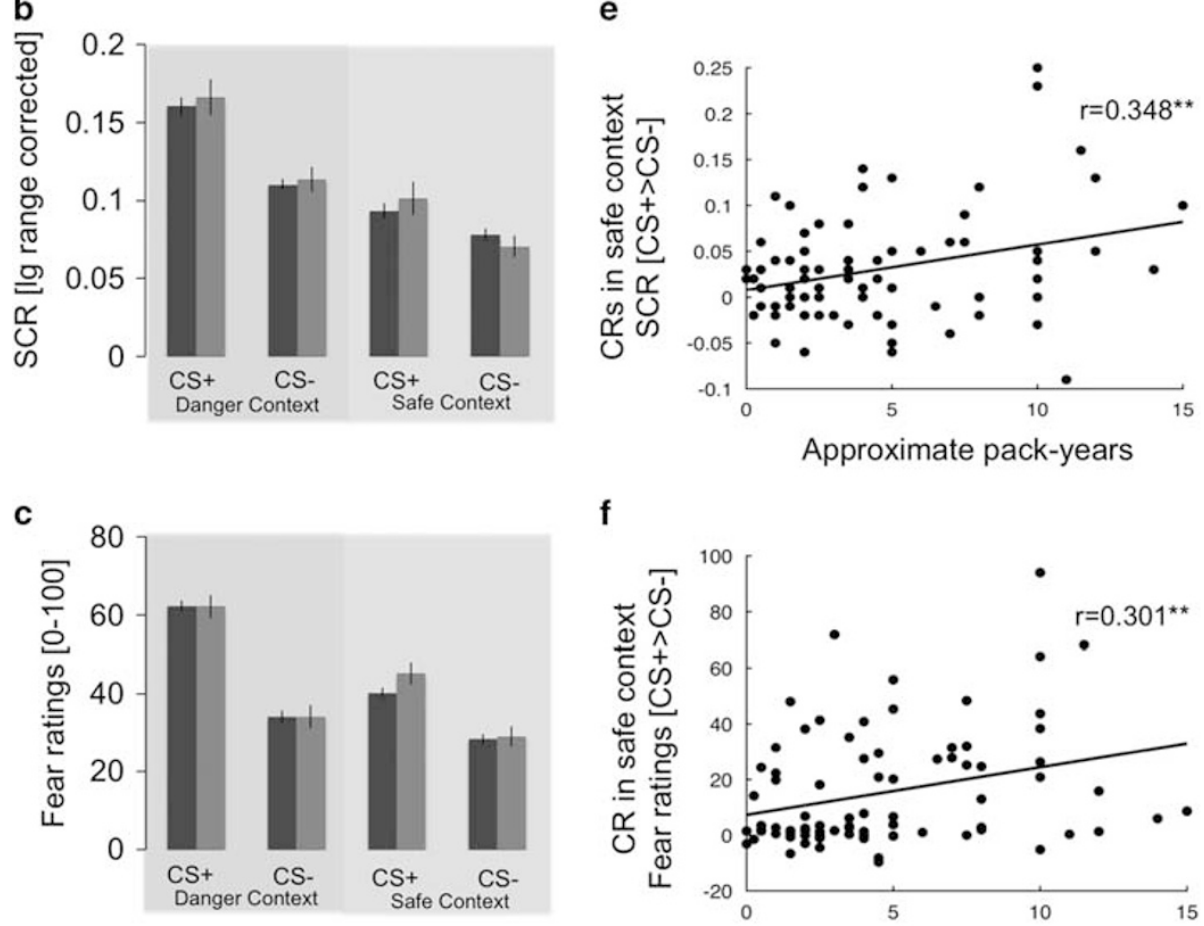

f
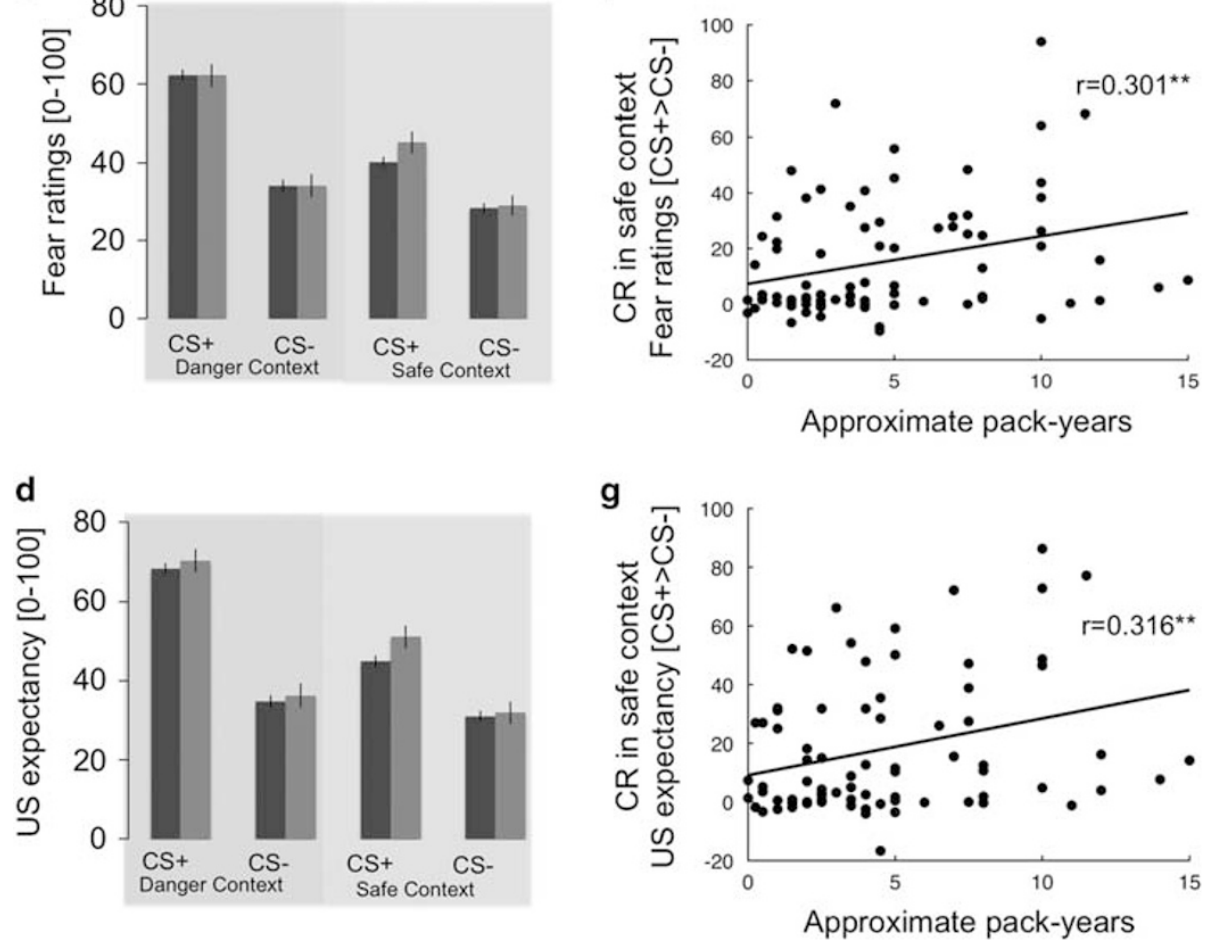

Figure I (a) Schematic illustration of the context-dependent cue conditioning and extinction paradigm, consisting of a learning phase (day I) and a retrieval phase $24 \mathrm{~h}$ later (day 2). (b) CS specific responses in non-smoking and smoking participants during day I (learning) in the danger and safe contexts measured as skin conductance responses (SCRs), (c) subjective fear and (d) US expectancy. (e) Individual amount of cigarette consumption (pack-years) is correlated with conditioned fear responses (CRs) in the safe context during learning on day I measured as SCRs, (f) subjective fear and (g) US expectancy.

reactions in PTSD patients. Fear reactions to traumaassociated stimuli after trauma and the frequent relapse following exposure-based treatments strongly rely on deficient learning and/or memory about the safety of everyday contexts (Vervliet et al, 2013). However, how smoking is associated with fear inhibition in humans has not been studied, yet.

We employed a cued fear conditioning and extinction paradigm (day 1). Twenty-four hours later (retrieval phase, day 2), CSs were presented again both in the conditioning ('danger') and extinction ('safe') contexts (Haaker et al, 2015b) (Figure 1a). We hypothesized impaired inhibition of CRs in the safe context in smokers relative to non-smokers.

\section{MATERIALS AND METHODS}

\section{Participants}

In total, 376 healthy individuals (mean age: 24.5 years, SD: 3.3; 258 females) participated in the study that was part of a large behavioral test-battery. 349 participants (242 females, $22 \%$ smokers) had valid data on both consecutive days of the 
Table I Sample Description of Smoking and Non-Smoking Participants

\begin{tabular}{|c|c|c|c|}
\hline Variable & $\begin{array}{l}\text { Mean (SD) } \\
\text { Non-smokers }\end{array}$ & $\begin{array}{l}\text { Mean (SD) } \\
\text { Smokers }\end{array}$ & $p$-value \\
\hline Sample size & 270 & 76 & - \\
\hline \multirow[t]{2}{*}{ Gender } & Females: $|8|$ & Females: 61 & $0.033^{b}$ \\
\hline & Males: 89 & Males: 15 & \\
\hline $1-10$ cigarettes & & $N=56(74.7 \%)$ & \\
\hline II-20 cigarettes & & $\begin{array}{c}N=19(25.3 \%) \\
\quad \text { Missing }=1\end{array}$ & \\
\hline $\begin{array}{l}\text { Approximate pack-years (one pack-year equals smoking II-20 } \\
\text { cigarettes per day for one year) }\end{array}$ & - & $4.73(3.50)$ & - \\
\hline School education & & & $0.45^{\mathrm{b}}$ \\
\hline High school degree ('Mittlere Reife' or equivalent) & $(N=2,0.7 \%)$ & $(N=2,2.6 \%)$ & \\
\hline Attended college ('Gymnasium') & $(N=3,1.1 \%)$ & $(N=0)$ & \\
\hline College degree ('Abitur') & $(N=12,4.4 \%)$ & $(N=1,1.3 \%)$ & \\
\hline Attended/attending university & $(N=198,73.3 \%)$ & $(N=56,73.7 \%)$ & \\
\hline University degree & $\begin{array}{l}(N=53,19.6 \%) \\
\text { Missing: } N=2\end{array}$ & $\begin{array}{l}(N=17,22.4 \%) \\
\text { Missing: } N=0\end{array}$ & \\
\hline STAI trait anxiety (sum score) & $36.49(8.23)$ & $38.05(9.15)$ & $0.183^{\mathrm{a}}$ \\
\hline $\mathrm{TCl}$ novelty seeking (sum score) & $20.76(5.90)$ & $23.28(6.23)$ & $0.00 I^{a}$ \\
\hline $\mathrm{TCl}$ reward dependence (sum score) & $16.10(4.17)$ & $|6.5|(4.07)$ & $0.439^{\mathrm{a}}$ \\
\hline
\end{tabular}

Sample description of smoking and non-smoking participants. Significant differences are marked bold. Controlling for individual differences in personality traits (STAl trait anxiety, TCl novelty seeking and TCl reward dependence), alcohol consumption, age, or gender did not substantially change the results in any dependent measure (context*smoking status interaction p's $<0.02$ in all analyses).

anpaired $t$-test, two-sided.

${ }^{b} \chi^{2}$ test exact, two-sided.

experiment and were included in the analyses. As such, the participants were not matched regarding smoking status and other individual differences, but rather to represent an age-specific proportion of smokers and non-smokers in Germany (Bundeszentrale für gesundheitliche Aufklärung, 2015). The effects of gender and trait anxiety in this sample have been published elsewhere (Haaker et al, 2015b; Lonsdorf et al, 2015) and current analyses controlled for their influence. Participants gave written informed consent. The study was approved by the local ethics committee in Hamburg (Ärztekammer Hamburg). Overall, 22\% of the participants were smokers and were allowed to smoke freely before the experiment on both days (see Table 1 for sample description and (Haaker et al, 2015b) for sample and task details).

\section{Stimuli}

Two geometric symbols (circle and triangle) served as CSs presented on either a blue or yellow screen background, which served as contexts. Assignment of the symbols and the background colors were counterbalanced across participants. Stimuli were presented using Presentation Software
(NeuroBehavioral Systems, Albany California, USA). The US consisted of a train of three square-wave pulses of $2 \mathrm{~ms}$ each (DS7A, Digitimer, Welwyn Garden City, UK) delivered on the right dorsal hand and was individually adjusted to a level of maximum tolerable pain (subjective ratings from 0: 'I feel nothing' to 10: 'maximally unpleasant', see Table 1) before the experiment. There were no difference between smoking and non-smoking participants with regard to the US current amplitude on day 1 (non-smokers mean: $4.3 \mathrm{~mA}$, SD: 5.7; smokers mean: $4.4 \mathrm{~mA}, \mathrm{SD}: 3.70, p>0.97$ ) or day 2 (non-smokers mean: $4.4 \mathrm{~mA}$, SD 5.71; smokers mean: $4.5 \mathrm{~mA}$ SD: $3.68, p>0.81$ ), and importantly there were no differences between groups in the subjective rating of the painfulness of the US on either day (day 1: non-smokers mean: 6.12, SD: 1.30 ; smokers mean: 6.16 , SD: $1.23, p>0.81$; day 2: non-smokers mean: 6.01, SD: 1.39; smokers mean: 6.03, SD: $1.23, p>0.92)$.

\section{Paradigm}

Fear conditioning and extinction learning was employed as a within-subject design (Figure 1a). The protocol consisted of an $A_{1} B_{1} A_{2} B_{2}$ design, with two interleaved blocks of fear 
conditioning in one context ( $\mathrm{A}_{1}$ and $\mathrm{A}_{2}$ : danger context) and extinction in another context $\left(\mathrm{B}_{1}\right.$ and $\mathrm{B}_{2}$ : safe context) on day 1 (learning). Each block consisted of 12 presentations of the $\mathrm{CS}+$ and the CS - , and each CS had a duration of $3 \mathrm{~s}$. The intertrial interval (ITI) was jittered in duration between 2.5 and $4 \mathrm{~s}$. During conditioning in the danger context, the CS+ was paired in $50 \%$ of trials with the aversive, electrotactile US (onset $2.5 \mathrm{~s}$ after CS+ onset), whereas the CS - was never paired with the US. During extinction in the safe context, both CSs were never paired with a US. Hence, on day 1, the $\mathrm{CR}$ (differential response to the $\mathrm{CS}+>\mathrm{CS}-$ ) in the danger context on day 1 can be used to index learned fear, whereas the $\mathrm{CR}$ in the safe context provides an inverse measurement of fear inhibition, a high CR indicating a deficit in inhibition (in particular, inhibition of fear to the CS+).

On day 2 (retrieval), US intensity was individually adjusted again. This was followed by 10 interleaved blocks of each context employing an $\mathrm{A}_{1} \mathrm{~B}_{1} \mathrm{~A}_{2} \mathrm{~B}_{2} \ldots \mathrm{A}_{10} \mathrm{~B}_{10}$ design. Each block consisted of one presentation of both the CS+ and the CS - , but without any US presentation in both contexts. Producing a CR to a CS+ in the danger context ('renewal', Bouton, 2002; Vervliet et al, 2013) can be considered adaptive, given the danger context has been associated with threat on day 1 . However, in the safe context, a high CR would indicate a deficit in the retrieval or expression of the fear-inhibitory memory formed during extinction in this context on day 1 . Hence, an adaptive response profile on day 1 would consist in a marked differentiation of CRs as a function of context (high in danger, low in safe). We term this 'contextual fear inhibition'.

\section{Physiological Measurements}

Skin conductance responses (SCRs) were recorded as previously described (Haaker et al, 2015b). In short, skin conductance was recorded using a BIOPAC MP35 amplifier (BIOPAC Systems, California, USA) and self-adhesive $\mathrm{Ag} / \mathrm{AgCl}$ electrodes placed on the palmar side of the left hand on the distal and proximal hypothenar eminence. Data were down-sampled to $10 \mathrm{~Hz}$, phasic SCRs were automatically scored as an increase in skin conductance within 1-3.5 s after stimulus onset and raw SCR amplitudes were then logarithmized and range-corrected [(SCR/SCRmax $)+1]$. Non-responders were defined as participants not showing any US response during the first half of the experiment (day 1) and excluded from all SCR analyses $(N=32)$. Additional 24 participants were excluded from the analyses, due to missing data on one of the days. In total 290 participants were included in the SCR analyses (61 smokers). Several control analyses (see previous publication, Haaker et al, 2015b) confirmed that USs that preceded or followed the CS presentations did not bias SCRs on day 1.

\section{Subjective Measurements}

In addition, participants rated their stress/fear/tension evoked by the CSs as well as their US expectancy after each block (4CS+ and 4CS - trials) on day 1 and after each block (1CS+ and 1CS - trials) on day 2, by answering the questions 'When you saw the symbol, how strong was your stress/fear/ tension' (for fear) and 'When you saw the symbol, to what extent did you expect the painful stimulus?' (for US expectancy). Ratings were given on a visual analog scale ranging from 0 (none) to 100 (maximum).

\section{Statistical Analyses}

In general, only participants with valid data-points on day 1 and day 2 were included in our analyses (Fear ratings and US expectancy: $N=349,242$ females, $22 \%$ smokers; SCR: $N=290$, 203 females, 21\% smokers). CRs were analyzed using repeated-measures ANOVAs with stimulus $(\mathrm{CS}+/ \mathrm{CS}-)$ and context (danger/safety) as within-subject factors and smoking status as between-subject factor, separately for each day. Repeated-measures ANOVAs controlling for individual differences in novelty seeking, consumption of alcohol and coffee, as well as age and gender and anxious temperament (STAI trait anxiety) were performed separately. Pack-years approximation was calculated as the product of self-reported daily cigarette consumption (1-10 cigarettes $=0.5$ packs per day; $11-20$ cigarettes $=1$ pack per day; $21-30$ cigarettes $=$ 1.5 packs per day) and the smoking duration (in years). Correlation between pack-years and CRs in both contexts were calculated as Pearson correlation coefficients and tested for statistical difference (Lee and Preacher, n.d.). Analyses controlling for the influence of individual differences in novelty seeking, consumption of alcohol and coffee, as well as age, gender and anxious temperament (STAI trait anxiety) were performed as linear regression models (step-wise inclusion) for day 1 and day 2. In addition, analyses exploring the moderation of smoking effects by trait anxiety were performed as linear regression models including pack-years (for day 1) or smoking status (for day 2), trait anxiety as well as the interaction of both (mean centered).

If appropriate, Greenhouse-Geisser corrections were adopted and $p<0.05$ was considered significant.

\section{RESULTS}

\section{Effects of Smoking on Day 1 (Learning)}

On day 1, no significant differences between smokers and non-smokers were observed during either conditioning (danger context) or extinction (safe context) (no main effects of or interaction with smoking status, all $p$ 's $>0.19$, see Figure $1 \mathrm{~b}-\mathrm{d}$ and Supplementary Table 1 for all main effects of task). However, CRs (CS+ $>\mathrm{CS}-$ ) in all dependent measurements were significantly correlated with pack-years in smokers in the safe context (SCR: $r=0.348, p=0.004$; fear ratings: $r=0.301, p=0.009$; US expectancy: $r=0.316$, $p=0.006$; Figure $1 \mathrm{e}-\mathrm{g}$ ), but not in the danger context (SCR: $r=0.129, p=0.30$; fear ratings: $r=0.196, p=0.094$; US expectancy: $r=0.123, p=0.12$ ). The correlation in the safe context was significantly stronger as compared with the danger context for the SCRs $(Z=-1.66$; $p$ (one-tailed) $=$ $0.049)$ and US expectancy $(Z=-1.89$; $p$ (one-tailed $)=0.029)$. This might suggest that chronic smoking is associated with enhanced CRs during extinction learning, pointing towards a fear inhibition deficit.

The groups of smoking and non-smoking participants differed with respect to gender distribution, alcohol consumption, coffee consumption and novelty seeking (Table 1).

Control analyses that included these variables, as well as trait anxiety, as predictors in a step-wise regression of CRs 
in the safe context on day 1 consistently revealed pack-years as the significant predictor across measurements (Supplementary Table 2).

Previous results within this sample (Haaker et al, 2015b) have revealed that inhibition of fear responses is associated with anxious temperament (trait anxiety). We tested if anxious temperament moderates the effect of pack-years on CRs in the danger and safety contexts on day 1 . Indeed, the step-wise regression model revealed that the interaction between pack-years and trait anxiety was a stronger predictor for the CR in the safe context as compared with each variable alone (variable main effects), across measurements (Supplementary Table 3 and Supplementary Figure 1). This might suggests that the influence of chronic smoking on fear inhibition during extinction learning increases with increased trait anxiety.

\section{Effects of Smoking on Day 2 (Retrieval)}

At the retrieval test (day 2), a context ${ }^{*}$ smoking status interaction was observed in all dependent measurements (SCRs: $\mathrm{F}(1,289)=8.50, p=0.004$, eta ${ }^{2}=0.03$; fear ratings: $\mathrm{F}(1,344)=9.15, \quad p=0.003, \quad$ eta $^{2}=0.03 ; \quad$ US expectancy: $\mathrm{F}(1,344)=6.47, p=0.01$, eta ${ }^{2}=0.02$; Figure $\left.2 \mathrm{a}-\mathrm{c}\right)$, in the absence of a main effect of smoking status $(p>0.25$, see Supplementary Table 4 for main effects of task). This interaction resulted from attenuated contextual inhibition of CRs in the safe relative to the danger context in smokers, as compared with non-smokers, irrespective of CS type (see Figure $2 \mathrm{~d}-\mathrm{f}$ for post-hoc tests). There were no correlations with pack-years (all $p>0.1$ ).

Control analyses that included gender distribution, alcohol consumption, coffee consumption and novelty seeking, as well as trait anxiety, as predictors in a step-wise regression of contextual inhibition on day 2 consistently revealed smoking status as the significant predictor across measurements (Supplementary Table 5). In addition, inclusion of these variables as separate covariates still yielded robust smoking status by context interactions across all measurements on day 2 (Supplementary Table 6). Importantly, the effect of smoking status on contextual inhibition on day 2 was not moderated by trait anxiety (Supplementary Table 7). Posthoc power calculation revealed that our sample size provided sufficient power to detect this effect (power $>0.99$; $G^{\star}$ Power Version 3.1.9.2).

\section{DISCUSSION}

Our results provide converging evidence from physiological responses and subjective evaluation that smokers as compared with non-smokers show impaired fear inhibition through the retrieval of contextual signals indicating safety. In addition, during extinction learning, the amount of smoking (pack-years) was associated with the level of conditioned fear responses. Participants with more packyears showed higher fear responses, in particular within a context that was safe (where the CS+ was no longer paired with the US). Interestingly, this effect during extinction learning was moderated by trait anxiety, which has previously been found to regulate inhibitory learning within this sample (Haaker et al, 2015b). By contrast, one day later, inhibition of fear memory in the safe context was impaired in smokers as compared with non-smokers regardless of the amount of cigarette consumption and independent of trait anxiety. Hence, during learning as well as during memory retrieval, smokers showed a deficit in contextual gating of safety, which mirrors the impairment found in clinical populations diagnosed with PTSD and anxiety disorders. Interestingly, we did not observe uniform effects of smoking on learning and retrieval processes. The amount of smoking (measured in pack-years) was correlated to a deficit in extinction learning. On the other hand, retrieval of safety memory was impaired in smoking individuals, despite the amount of pack-years. This might suggest slightly different mechanisms of how smoking impairs learning, consolidation and retrieval of fear and safety.

However, within the framework of RDoc, we provide evidence that smoking is associated with deficits in the negative valence system in responses to acute threat. Although this might explain the co-occurrence of smoking and PTSD diagnoses, we suggest that this association exists in undiagnosed populations, as well.

Our participants were allowed to smoke freely before the experiments on both days, and we did not measure smoking before the experiments or nicotine status during the experiments. However, the associations observed on day 1 with a measure of long-term cigarette consumption (packyears) indicate that our findings might be related to chronic rather than acute smoking effects.

Three mechanisms may contribute to these effects. First, being a smoker may interfere with memory consolidation or retrieval and/or related discrimination processes, possibly via nicotinergic or dopaminergic mechanisms (Fu et al, 2007; Kutlu and Gould, 2015; Subramaniyan and Dani, 2015). Although several studies found alterations of dopaminergic functioning in smokers (Bloomfield et al, 2014; Cosgrove et al, 2015; Rademacher et al, 2015), there is evidence that dopamine is involved in the consolidation of safety memories to inhibit the return of fear responses (Haaker et al, 2013, 2015a). Second, maintained smoking habits (Winkler et al, 2011) and a persistent conditioned fear response in safe contexts might be due to an common underling deficit in flexibly adjusting learned associations. Third, trait anxiety might moderate the effect of smoking on fear inhibition deficits.

Previous studies examining the association between PTSD and smoking found evidence for bi-directional influence of smoking and development of PTSD (Breslau et al, 2004), albeit less evidence for shared genetic heritability that might explain a common underlying deficit of both (Fu et al, 2007; Koenen et al, 2005, 2006).

Our results have several limitations. Participants in this study were not matched for several individual differences (eg, gender) between smoking and non-smoking individuals. Moreover, our analysis relied on self-report of smoking status and pack-years. In particular the measurement of pack-years might not be optimal to access reliable information about chronicity of nicotine consumption. Hence, imprecisely measured confounders or the presence of unmeasured confounders cannot be ruled out in our results. Future studies will be important to replicate our findings in matched groups, using biochemical examination of smoking status (eg, levels of CO or metabolites as cotinine) and testing 


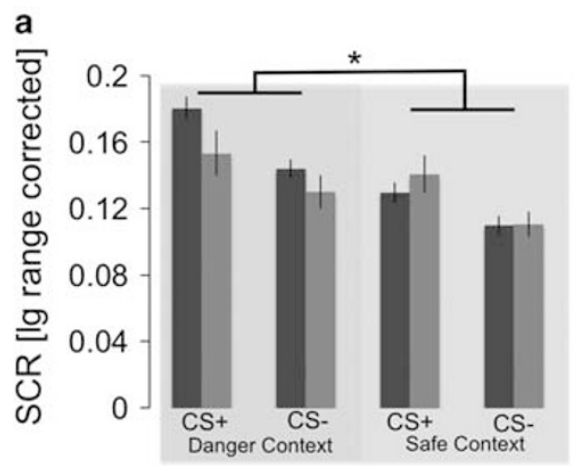

d

b

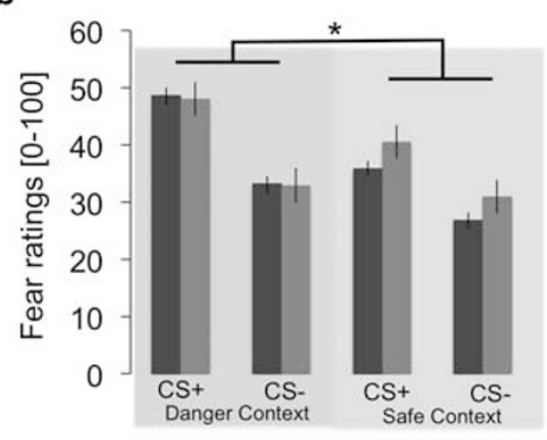

C

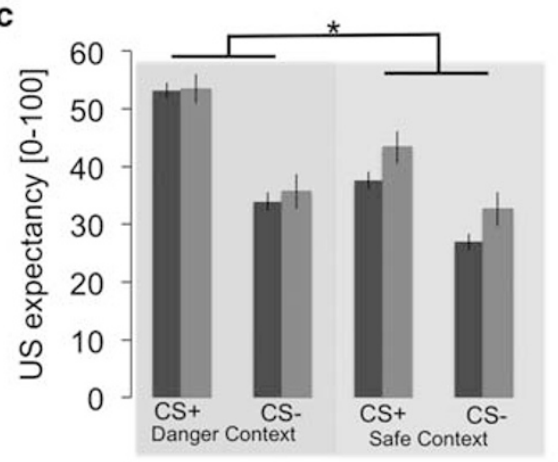

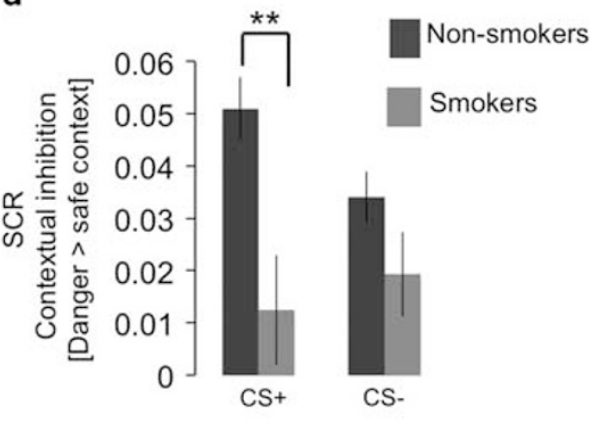

e

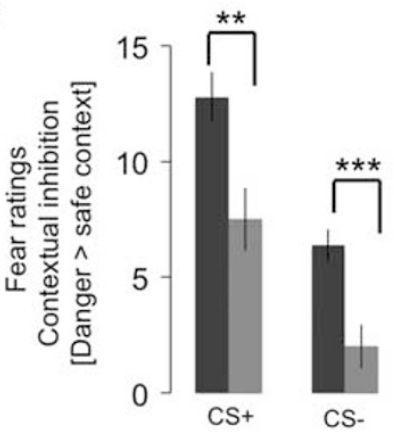

f

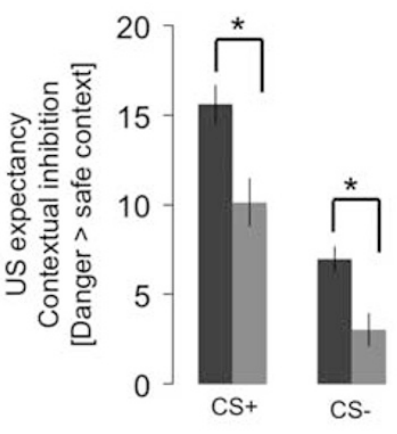

Figure 2 (a) CS responses during day 2 (retrieval) reveal a context*smoking status (non-smoking vs smoking participants) interaction measured as skin conductance responses (SCRs), (b) subjective fear and (c) US expectancy. (d) During memory retrieval on day 2, smokers show less contextual inhibition of SCRs (CS+: $t(346)=3.07, p=0.002 ; C S-: t(346)=1.54, p=0.14)$, (e) subjective fear $(C S+: t(346)=3.04, p=0.003 ; C S-: t(346)=3.76, p<0.00 \mathrm{I})$ and $(f)$ US expectancy $(C S+: t(346)=2.56, p=0.012 ; C S-: t(346)=2.46, p=0.015)$. $* p<0.05 ; * *=p<0.01$; $* * * *<<0.001$. Error bars represent the standard error of the mean.

the effect of instructed abstinence. In addition, pharmacological studies testing the causal effect of nicotine, might help to understand the neuropharmacological mechanisms in more depth.

Although our study cannot provide causal mechanisms that explain the association between smoking and impaired fear inhibition, it might well be that influences of smoking on broader processes involved in learning, eg, attention (Ashare and Hawk, 2012; Lerman et al, 2001) have contributed to our results. Nevertheless, the effects observed here underline the fact that co-occurrence of smoking and deficits in fear inhibition are prevalent in undiagnosed populations. Moreover, this deficit in fear inhibition is due to impaired identification of contextual safety, in line with previous experiments of nicotine exposure in rodents (Kutlu et al, 2014; Kutlu and Gould, 2014).

Importantly, deficient inhibition of fear responses through contextual safety signals is a hallmark of PTSD and anxiety- related disorders (Vervliet et al, 2013). Hence, our results suggest for the first time contextual inhibition of fear as a potential mechanistic link between smoking and the development and maintenance of these disorders. Prospective investigations are warranted to unravel causal relationships.

\section{FUNDING AND DISCLOSURE}

The funding sources had no involvement in study design; in the collection, analysis, and interpretation of data; in the writing of the report; and in the decision to submit the paper for publication. The authors declare no conflict of interest.

\section{ACKNOWLEDGMENTS}

This work was supported by a State of Hamburg excellence initiative (Landesexzellenzcluster 12/09 'neurodapt'), the 
Deutsche Forschungsgemeinschaft (Grants KA 1623/3-1, KA 1623/4-1, SFB1193 subprojects B01 and C01), and the State of Rhineland-Palatinate's Focus Program Translational Neuroscience (FTN).

\section{REFERENCES}

Ashare RL, Hawk LW (2012). Effects of smoking abstinence on impulsive behavior among smokers high and low in ADHD-like symptoms. Psychopharmacology 219: 537-547.

Bloomfield MAP, Pepper F, Egerton A, Demjaha A, Tomasi G, Mouchlianitis E et al (2014). Dopamine function in cigarette smokers: an $\left[{ }^{18} \mathrm{~F}\right]-\mathrm{DOPA}$ PET study. Neuropsychopharmacology 39: 2397-2404.

Bouton ME (2002). Context, ambiguity, and unlearning: sources of relapse after behavioral extinction. Biol Psychiatry 52: 976.

Breslau N, Novak SP, Kessler RC (2004). Psychiatric disorders and stages of smoking. Biol Psychiatry 55: 69-76.

Bundeszentrale für gesundheitliche Aufklärung (2015). Die Drogenaffinität Jugendlicher in der Bundesrepublik Deutschland 2015. Available at < http://www.bzga.de/forschung/studien-unter suchungen/studien/suchtpraevention/?sub $=100>$.

Cook B, Wayne G, Kafali E, Liu Z, Shu C, Flores M (2014). TRends in smoking among adults with mental illness and association between mental health treatment and smoking cessation. JAMA 311: 172-182.

Cosgrove KP, Esterlis I, Sandiego C, Petrulli R, Morris ED (2015). Imaging tobacco smoking with PET and SPECT. Curr Top Behav Neurosci 24: 1-17.

Cuthbert BN, Insel TR (2013). Toward the future of psychiatric diagnosis: the seven pillars of RDoC. BMC Med 11: 126.

Fu SS, McFall M, Saxon AJ, Beckham JC, Carmody TP, Baker DG et al (2007). Post-traumatic stress disorder and smoking: a systematic review. Nicotine Tob Res 9: 1071-1084.

Haaker J, Gaburro S, Sah A, Gartmann N, Lonsdorf TB, Meier K et al (2013). Single dose of L-dopa makes extinction memories context-independent and prevents the return of fear. Proc Natl Acad Sci USA 110: E2428-E2436.

Haaker J, Lonsdorf TB, Kalisch R (2015a). Effects of post-extinction l-DOPA administration on the spontaneous recovery and reinstatement of fear in a human fMRI study. Eur Neuropsychopharmacol 25: 1544-1555.

Haaker J, Lonsdorf TB, Schümann D, Menz M, Brassen S, Bunzeck $\mathrm{N}$ et al (2015b). Deficient inhibitory processing in trait anxiety: Evidence from context-dependent fear learning, extinction recall and renewal. Biol Psychol 111: 65-72.

Isensee B, Wittchen H-U, Stein MB, Höfler M, Lieb R (2003). Smoking increases the risk of panic: findings from a prospective community study. Arch Gen Psychiatry 60: 692-700.
Kirby AC, Hertzberg BP, Collie CF, Yeatts B, Dennis MF, McDonald SD et al (2008). Smoking in help-seeking veterans with PTSD returning from Afghanistan and Iraq. Addict Behav 33: $1448-1453$.

Koenen KC, Hitsman B, Lyons MJ, Niaura R, McCaffery J, Goldberg J et al (2005). A twin registry study of the relationship between posttraumatic stress disorder and nicotine dependence in men. Arch Gen Psychiatry 62: 1258-1265.

Koenen KC, Hitsman B, Lyons MJ, Stroud L, Niaura R, McCaffery J et al (2006). Posttraumatic stress disorder and late-onset smoking in the Vietnam era twin registry. J Consult Clin Psychol 74: 186-190.

Kutlu MG, Gould TJ (2014). Acute nicotine delays extinction of contextual fear in mice. Behav Brain Res 263: 133-137.

Kutlu MG, Gould TJ (2015). Nicotine modulation of fear memories and anxiety: Implications for learning and anxiety disorders. Biochem Pharmacol 97: 498-511.

Kutlu MG, Oliver C, Gould TJ (2014). The effects of acute nicotine on contextual safety discrimination. J Psychopharmacol 28: 1064-1070.

Lasser K, Boyd J, Woolhandler S, Himmelstein DU, McCormick D, Bor DH (2000). Smoking and mental illness: a population-based prevalence study. JAMA 284: 2606-2610.

Lee IA, Preacher KJ. Calculation for the test of the difference between two dependent correlations with one variable in common [computer software]. Available at http://quantpsy.org.

Lerman C, Audrain J, Tercyak K, Hawk LW, Bush A, Crystal-Mansour S et al (2001). Attention-deficit hyperactivity disorder (ADHD) symptoms and smoking patterns among participants in a smoking-cessation program. Nicotine Tob Res 3: 353-359.

Lonsdorf TB, Haaker J, Schümann D, Sommer T, Bayer J, Brassen S et al (2015). Sex differences in conditioned stimulus discrimination during context-dependent fear learning and its retrieval in humans: the role of biological sex, contraceptives and menstrual cycle phases. J Psychiatry Neurosci 40: 140336.

Rademacher L, Prinz S, Winz O, Henkel K, Dietrich CA, Schmaljohann J et al (2015). Effects of smoking cessation on presynaptic dopamine function of addicted male smokers. Biol Psychiatry 80: 198-206.

Subramaniyan M, Dani JA (2015). Dopaminergic and cholinergic learning mechanisms in nicotine addiction. Ann N Y Acad Sci 1349: 46-63.

Taylor JH, Jakubovski E, Bloch MH (2015). Predictors of anxiety recurrence in the Coordinated Anxiety Learning and Management (CALM) trial. J Psychiatr Res 65: 154-165.

Vervliet B, Craske MG, Hermans D (2013). Fear extinction and relapse: state of the art. Annu Rev Clin Psychol 9: 215-248.

Winkler MH, Weyers P, Mucha RF, Stippekohl B, Stark R, Pauli P (2011). Conditioned cues for smoking elicit preparatory responses in healthy smokers. Psychopharmacology 213: 781-789.

Supplementary Information accompanies the paper on the Neuropsychopharmacology website (http://www.nature.com/npp) 